\title{
Polydopamine-assisted functionalization of heparin and vancomycin onto microarc-oxidized 3D printed porous Ti6Al4V for improved hemocompatibility, osteogenic and anti-infection potencies
}

Teng Zhang ${ }^{1,2}$, Wenhao Zhou ${ }^{3}$, Zhaojun $\mathrm{Jia}^{3,4}$, Qingguang Wei ${ }^{1,2}$, Daoyang Fan ${ }^{1,2}$, Jianglong Yan $^{3}$, Chuan Yin ${ }^{1,2}$, Yan Cheng ${ }^{3}$, Hong Cai ${ }^{1,2}$, Xiaoguang Liu ${ }^{1,2}$, Hua Zhou ${ }^{1,2}$, Xiaojie Yang ${ }^{5}$, Yufeng Zheng ${ }^{3,4^{*}}$ and Zhongjun Liu ${ }^{1,2^{*}}$

\begin{abstract}
Enhanced antiinfection activities, improved hemocompatibility and osteo-compatibility, and reinforced osseointegration are among the most important considerations in designing multifunctional orthopedic biomaterials. Hereby, anti-infective and osteogenic multifunctional 3D printed porous Ti6Al4V implant with excellent hemocompatibility was successfully designed and fabricated. In brief, osteogenic micro-arc oxidation (MAO) coatings with micro/nanoscale porous topography were generated in situ on 3D printed Ti6Al4V scaffolds, on which heparin and vancomycin were easily immobilized. The surface microstructure, morphology, and chemical compositions were characterized employing scanning electron microscopy (SEM), X-ray photoelectron spectroscopy (XPS) and Fourier transform infrared spectroscopy (FTIR). High loading capacity and sustained vancomycin release profiles were revealed using high performance liquid chromatography (HPLC). Favorable antibacterial and antibiofilm performances against pathogenic Staphylococcus aureus (S. aureus) were validated in vitro through microbial viability assays, Live/Dead bacterial staining, and crystal violet staining. Human mesenchymal stem cells (hMSCs) were seeded on the scaffolds and their proliferation and viability were assessed using Cell Counting Kit and Live/Dead cell viability kit. Further, osteoblastic differentiation abilities were evaluated using alkaline phosphatase (ALP) activity as a hall marker. Additionally, the improved
\end{abstract}

hemocompatibility of the heparinized scaffolds was confirmed by activated partial thromboplastin time (APTT), prothrombin time (PT) and thrombin time (TT). Overall, our results show that the surface-modified $3 \mathrm{D}$ printed porous Ti6Al4V possesses balanced antibacterial and osteogenic functions while exhibiting extra anticlotting effects, boding well for future application in customized functional reconstruction of intricate bone defects.

Keywords: 3D printing, porous Ti6Al4V, anti-infection, microarc oxidation, osseointegration, vancomycin

\section{INTRODUCTION}

Diseases, traumas, and the elevated life expectation from the global aging population are continuing to stimulate the demand for advanced custom implants that renovate or enhance impaired tissue functions. Because of their excellent mechanical properties, good biocompatibility and appropriate costs, titanium and its alloys remain one of the most popular biomaterials in building orthopedic implants and devices for such applications in load-bearing anatomic locations such as hip/knee joints. A recent technical innovation in the fabrication of titanium biomaterials is $3 \mathrm{D}$ printing, such as the electron beam melting (EBM) and laser beam melting (LBM) [1]. It has enabled the layer-by-layer creation of designer custom-

\footnotetext{
${ }^{1}$ Department of Orthopedics, Peking University Third Hospital, Beijing 100191, China

${ }^{2}$ Beijing Key Laboratory of Spinal Diseases, Beijing 100191, China

${ }^{3}$ Center for Biomedical Materials and Tissue Engineering, Academy for Advanced Interdisciplinary Studies, Peking University, Beijing 100871, China

${ }^{4}$ Department of Materials Science and Engineering, College of Engineering, Peking University, Beijing 100871, China

${ }^{5}$ Beijing AKEC Medical Company Ltd., Beijing 102200, China

* Corresponding authors (emails: liuzj@medmal.com.cn (Liu Z); yfzheng@pku.edu.cn (Zheng Y))
} 
made titanium scaffolds with geometrically complex and fully interconnected architecture, attracting extraordinary interest for restoring (some of) the skeleton integrity of critically defected bone tissues in animals and patients $[2,3]$. Implants as fabricated hold unparalled advantages, including but not limited to adjustable sizes, pores and shapes, greatly enlarged surface areas, tailored elastic modulus and mechanical strength, sufficient space for ingrowth of bone and blood vessel, and open channels facilitating nutrient supply $[2,4]$.

Poor osteointegration and peri-implant infections remain leading causes of implant loosing and failure. With respect to porous titanium, enhancement of bone ingrowth efficiency and biointegration performances and the prevention/fighting of biomedical device-associated infections (BAI) are two major keys to overcome implant looseness and ultimately establish stable functional implant/bone interfaces [4]. BAI is a prominent cause of clinical implant failure whose occurrences range averagely between $2 \%$ and $5 \%$ and can reach up to $30 \%$ in the cases of open fractures [5]. The handle of an infected implant is notoriously excruciating, expensive, and time consuming [6]. In addition, deep vein thrombosis, inflammation and pulmonary embolism, originating primarily from the relatively inferior hemocompatibility of titanium, are problematic complications after surgery intervention that can also affect the eventual success of implantation [7], and this is especially true for the elderly patients and those with a history of venous thromboembolism. The osteointegration issue can be addressed through tailoring porous macro-architectures and surface properties. A plethora of earlier efforts were centered on optimizing titanium scaffolds' porosity and geometrical structure [8], and this focus was later shifted towards the alteration of their surface topography, landscape and composition to better mimic that of natural boney extracellular matrix (ECM). Through the use of anodization, microarc oxidization (MAO), acid-alkali treatments and electrodeposition [9-11], prior researchers have successfully covered the entire surfaces of porous titanium with micro/nano topography and/or bioactive composition, displaying appreciable osteogenic and osteointegration effects [12]. Particularly, MAO is a simple and cheap technique that warrants adoption for clinical use; it is capable of conferring both micro/nanotopographical features (biophysical cues) and biochemical cues to metallic objects of complex shapes [13]. In our previous study, MAO was employed to grow in situ micro/nanoporous $\mathrm{TiO}_{2} / \mathrm{CaP}$ coatings on $3 \mathrm{D}$ printed porous Ti6Al4V, leading to excitingly promoted osseointegration via altered bone in-growth patterns and interlocked bone/ implant interface [10].

Our aim here is to further add anti-infection, and concomitantly, anti-thrombosis functionalities to the abovementioned $\mathrm{TiO}_{2} / \mathrm{CaP}$-coated hierarchical porous titanium, rendering it capable of combating bacterial biofilm formation and inhibiting thrombosis as well. Regardless of various anti-infective and osteogenic coatings, such as the plasma spraying coating and layer-bylayer (LBL) coatings $[14,15]$, are invented, many intricate issues are far beyond their capability to address, especially the porous topography characteristic of the $3 \mathrm{D}$ printed Ti6Al4V and the practicability issue. There also exist studies incorporating Ag to hydroxyapatite/titania coatings to get anti-infective function [16]. Once becoming sessile and adhered, bacteria can readily multiply to colonize the biomaterial surface and initiate impenetrable biofilms, eventually producing recalcitrant infections [17]. It is evident that merely $10^{2}$ CFU Staphylococcus aureus (S. aureus) bacteria are potent to infect $95 \%$ subcutaneous implants in vivo [18]. Implant associated biofilms are highly tolerant to host immune responses and systemic antibiotic attacks. Systemic use of orders of magnitude higher antibiotic dosages are thus demanded to kill bacteria in a "biofilm mode of growth" compared to their planktonic counterparts, which however, cannot be tolerated by the human body [19]. Equipping implants with antibiotic-eluting coatings possessing active, localized delivery characteristics hence represents a critical parallel strategy. Such a strategy is expected to create a strong antimicrobial microenvironment in the immediate as-threatened vicinity of an implant while circumventing injuriously high drug concentrations elsewhere in the human. In the current study, we elaborately functionalized $\mathrm{TiO}_{2} / \mathrm{CaP}$-coated hierarchical porous titanium biomaterials with a clinically powerful glycopeptide antibiotic vancomycin (Van) through a convenient polydopamine (PD)/heparin (Hep) assisted coating route (Fig. 1), anticipating extremely high drug loading capacity and tailored release kinetics.

Heparin, a versatile highly-sulfated natural polysaccharide, was employed as a charging vehicle of vancomycin. To date, heparin-functionalized biomaterial systems have displayed significant merits for loading and controlled release of collagen, antibiotics, growth factors (e.g., VEGF, $\beta$-FGF, TGF- $\beta$ ) and other biomolecules due to the dynamic binding interplays (ionic interactions, hydrogen bonding, and hydrophobic forces, etc.) between heparin and these substances [20-25]. Importantly, as heparin has mutual solubility in common antibiotics (e.g., 

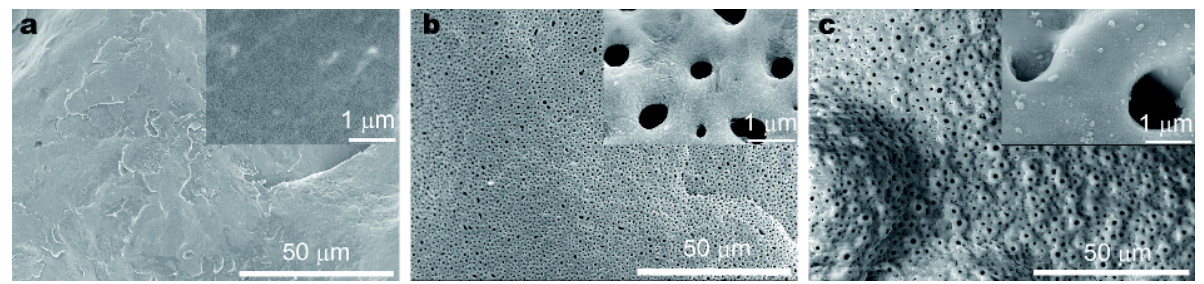

Figure 1 SEM images ( $\times 1000$ and $\times 5000$ magnification) of the surface landscapes of TS (a), TS-M (b) and TS-M/P/V (c) scaffolds.

vancomycin, gentamicin) and can interact actively with them [26-28], it has been applied as a viable antibiotic lock solution for long-term therapy of catheter-related blood stream infections. Meanwhile, heparin is a clinically popular anticoagulant drug, and materials with surface heparinization have demonstrated generally improved anti-inflammatory and anticoagulant properties and biocompatibility $[29,30]$. PD is another crucial component that mediates heparin immobilization onto porous titanium and imparted biofunctionalities to the implants. It is a self-polymerization product of a catechol-rich molecule "dopamine", as derived from mussel foot proteins [31]. Interestingly, dopamine is capable of adaptively forming crosslinked strongly adhesive PD nanolayer onto virtually any substrate of any material/ shape at alkaline conditions, proving an advantageous platform for diverse surface design of biomaterials $[32,33]$. PD is rich in reactive quinone, carboxy, amino, imine, and phenol groups [34]. In particular, because of the coupling between the amine groups of heparin and quinone species in $\mathrm{PD}$, heparin have been covalently immobilized onto various PD-modified substrates $[35,36]$.

Therefore, in our design, PD serves as an adherent anchor of heparin immobilized to the underlying $\mathrm{TiO}_{2} /$ $\mathrm{CaP}$ MAO layer while heparin acts as an anti-hrombosis agent and a potent carrier of vancomycin. Through the interaction of the above substances, an integral hybrid coating system (MAO-PD-Hep/Van) on titanium scaffolds that stores vancomycin molecules efficiently and liberates them sustainably is created. The successful functionalization of heparin and antibiotics onto the scaffold surface was at first verified by thorough material characterization and in vitro release studies. The antithrombogenic properties of the materials were estimated in vitro by the kinetic clotting experiment and platelet adhesion experiment. The antibacterial and anti-biofilm performances of the scaffolds were then investigated using S. aureus as pathogenic bacteria. Further, the cell culture responses (proliferation, viability, and osteo- differentiation activity) of the modified specimens towards human mesenchymal stem cells (hMSCs) were examined preliminarily for osteogenic applications.

\section{EXPERIMENTAL SECTION}

\section{Scaffold fabrication}

3D printed macroporous Ti6Al4V scaffolds $(\varnothing=10 \mathrm{~mm}$, $H=5 \mathrm{~mm}$ ) were designed by a computer assisted design (CAD) software (Magics, Materialise, Belgium) and fabricated layer by layer using EBM. The porous architecture was designed based on a dodecahedron unit cell with a pore size of $640 \mu \mathrm{m}$, strut diameter of $400 \mu \mathrm{m}$ and porosity of $73 \%$, which, as found in our previous study, is beneficial for the in-growth of bone and blood vessels [37]. All specimens were subjected to air blasting and successively washed in acetone, alcohol, deionized water in an ultrasonic cleaner for $20 \mathrm{~min}$ each and dried at $60^{\circ} \mathrm{C}$ overnight for use. For simplicity, hereafter the Ti6Al4V scaffolds are denoted as TS.

\section{Surface modification}

The MAO process was carried out on a JH-10 pulsing power supply (Jin-hu-lv-bao Co., Ltd., Beijing, China; frequency $500 \mathrm{~Hz}$, duty ratio $10 \%$ ) at a working voltage of $250 \mathrm{~V}$ for $3 \mathrm{~min}$. The nanoscale porous topography, especially the size and morphology of the pore, under different electric parameters was observed with scanning electron microscopy (SEM) and then we chose the best electric parameter (Supplementary information, Fig. S1). The porous TS was used as anode and a stainless plate as cathode, with a distance of $10 \mathrm{~cm}$ in between. The electrolyte was an aqueous solution of $0.065 \mathrm{~mol} \mathrm{~L}^{-1} \mathrm{Ca}$ $\left(\mathrm{CH}_{3} \mathrm{COO}\right)_{2} \cdot \mathrm{H}_{2} \mathrm{O}, 0.03 \mathrm{~mol} \mathrm{~L}^{-1} \mathrm{NaH}_{2} \mathrm{PO}_{4}, 0.065 \mathrm{~mol} \mathrm{~L}^{-1}$ EDTA-2Na, and $0.5 \mathrm{~mol} \mathrm{~L}^{-1} \mathrm{NaOH}$. For vancomycin immobilization, scaffolds were first soaked in $10 \mathrm{~mL}$ of freshly prepared dopamine hydrochloride solution $\left(2 \mathrm{mg} \mathrm{mL}^{-1}, \mathrm{pH}\right.$ adjusted to 8.5$)$ in $10 \mathrm{mmol} \mathrm{L}^{-1}$ Tris buffer for $24 \mathrm{~h}$ to form a PDA layer on the surfaces. Afterward, samples were oscillated in a mixed solution of 
vancomycin and heparin for $6 \mathrm{~h}$. Scaffolds after MAO, $\mathrm{PDA}$, and heparin/vancomycin treatments were denoted as TS-M, TS-M/P, TS-M/P/V, respectively. In addition, the scaffolds without MAO but identically treated by PDA and heparin/vancomycin were denoted as TS-P/V. The scaffold after MAO, PDA, and heparin treatments but without vancomycin was denoted as TS-M/P/H.

\section{Material characterization}

The surface morphology and cross-sectional features of the outer and inner surface of MAO treated scaffolds were characterized employing a field emission SEM (FESEM, S-4800, Hitachi). The chemical composition was identified using X-ray photoelectron spectroscopy (XPS, Kratos, UK). The functional groups in TS-M/P/V were analyzed on a Fourier transform infrared spectrometer (FTIR, ECTOR22, Nicolet, USA).

\section{In vitro drug release}

In vitro vancomycin release test of $\mathrm{TS}-\mathrm{M} / \mathrm{P} / \mathrm{V}$ and $\mathrm{TS}-\mathrm{P} / \mathrm{V}$ were done using high performance liquid chromatography (HPLC) analysis (HPLC on an Agilent 1200 series system equipped with a diode array detector DAD, Agilent Technologies). Three samples of each type were submersed in $2 \mathrm{~mL}$ of phosphate buffered saline (PBS, $\mathrm{pH}$ 7.4) in $10-\mathrm{mL}$ centrifuge tubes and incubated at room temperature under constant rotation (100 rpm) over a 21 day period, respectively. The PBS was removed and replaced by the same volume of fresh one at given time intervals. The mobile phase of the column was composed of methanol: $\mathrm{KH}_{2} \mathrm{PO}_{4}$ approximately 5:95, the flow rate of the mobile phase was $1.0 \mathrm{~mL} \mathrm{~min}^{-1}$, and the detection wavelength was $236 \mathrm{~nm}$. A calibration curve (concentration range $0-150 \mu \mathrm{g} \mathrm{mL}^{-1}, y=0.0043 x+0.0103, R^{2}=$ $0.9989, y$ represents OD value and $x$ represents the concentration of vancomycin) obtained with known vancomycin concentrations was adopted to determine the vancomycin concentrations in the PBS. Each test was repeated three times to ensure reproducibility of the results. In order to investigate the effect of MAO on the loading capacity of vancomycin, we also compared the release kinetics between TS-M/P/V and TS-P/V.

\section{Bacteria culture and inoculation}

Gram-positive $\left(\mathrm{G}^{+}\right)$S. aureus is one of the most common pathogens causing infections in implants. The bacteria were cultivated aseptically in Luria-Bertani (LB) nutrient broth at $37^{\circ} \mathrm{C}$ and $180 \mathrm{rpm}$ rotation for $12 \mathrm{~h}$, and then $500 \mu \mathrm{L}$ of the broth was taken out and added to $20 \mathrm{~mL}$ of $\mathrm{LB}$ medium for culturing in a $37^{\circ} \mathrm{C}$ shaker incubator at
$200 \mathrm{rpm}$ for $1 \mathrm{~h}$ to reach the exponential growth phase $\left(1 \times 10^{8} \mathrm{CFU} \mathrm{mL} \mathrm{L}^{-1}\right)$. For inoculation, the specimens $(n=3)$ were sterilized and immersed in $1 \mathrm{~mL}$ of broth containing bacteria at adjusted concentrations in 24-well plates. Cultivation was performed in a microbiological incubator at $37^{\circ} \mathrm{C}$ in a $>95 \%$ humidified atmosphere with $5 \% \mathrm{CO}_{2}$.

\section{In vitro antibacterial assays}

\section{MIC of vancomycin for loading}

To guarantee that the TS-M/P/V samples have sufficient antimicrobial activity, the minimum inhibitory concentration (MIC) of vancomycin for loading was determined by a micro-dilution method, against $S$. aureus. In brief, six sets of TS-M/P/V samples were prepared by oscillation in vancomycin with different concentrations, that is $10,20,30,40,50,60 \mu \mathrm{gL}^{-1}$. Each set of sample in triplicate was inoculated aseptically in the presence of $2 \mathrm{~mL}$ of $S$. aureus suspension (approximately $1 \times 10^{8} \mathrm{CFU}$ $\mathrm{mL}^{-1}$ ) at $37^{\circ} \mathrm{C}$ for $24 \mathrm{~h}$, and the solution turbidities were distinguished visually to determine the MIC value. The lowest drug loading concentration absolutely inhibiting bacterial growth was taken as the MIC and corresponding $\mathrm{TS}-\mathrm{M} / \mathrm{P} / \mathrm{V}$ was qualified for implant application. The MIC value of vancomycin for loading is $50 \mu \mathrm{g} \mathrm{mL}^{-1}$ and the corresponding TS-M/P/V is named TS-M/P/V $\left(50 \mu \mathrm{g} \mathrm{m}^{-1}\right)$ (Fig. S2).

\section{Antibacterial assays}

For antibacterial assays, TS, TS-M and TS-M/P/V $\left(50 \mu \mathrm{g} \mathrm{mL}^{-1}\right)$ samples were incubated respectively with $1 \mathrm{~mL}$ of bacterial suspensions at a concentration of $10^{8}$ $\mathrm{CFU} \mathrm{mL} \mathrm{m}^{-1}$ per well for 4 and $24 \mathrm{~h}$. The antibacterial rates (Ra) of different samples against planktonic (Rap) and adhered ( $R \mathrm{aa}$ ) bacteria were quantitatively evaluated using a Microbial Viability Assay Kit-WST (Dojindo, Japan) according to the manufacturer's directions, which can calorimetrically detect bacteria metabolic activities that correspond well with the living bacterial amounts [34]. Briefly, at the end of incubation, the remaining culture media were collected, and the scaffolds were rinsed thoroughly to remove the none-adherent bacteria. Then, the sample media and the scaffolds themselves were incubated respectively with LB containing 5\% WST for $2 \mathrm{~h}$, and absorbance at $450 \mathrm{~nm}\left(\mathrm{~A}_{450}\right)$ was read on a microplate reader (Bio-RAD, Hercules). The Rap and Raa were calculated by the following equations: Rap $(\%)=(A$ $-B) / A \times 100$; Raa $(\%)=(C-D) / C \times 100$, where $A, B$ are average $\mathrm{A}_{450}$ values of testing solutions for bacteria from collected media for TS and TS-M/P/V (or TS-M), while 
$C, D$ are those for adhered bacteria on the surfaces of TS and TS-M/P/V (or TS-M). In addition, the 4-h cultures were rinsed and fixed in 2.5\% glutaraldehyde and then dehydrated in a series of ethanol water mixture $(50 \%$, $70 \%, 95 \%$ and $100 \%$ ethanol) sequentially for $10 \mathrm{~min}$ respectively. The morphology of bacteria adhered on scaffolds was observed under SEM. Further, the viable and dead bacteria cells on samples at $24 \mathrm{~h}$ were distinguished by fluorescent staining using Live/Dead BacLight $^{\mathrm{TM}}$ Bacterial Viability Kits (Molecular Probes ${ }^{\mathrm{TM}}$, Invitrogen). Notably, the cell membranes of living bacteria were intact and stained green while dead ones with disrupted cell membranes were stained red.

\section{Anti-biofilm assay}

The anti-biofilm capacity of the scaffolds was assayed by exposing them to $S$. aureus suspension (approximately

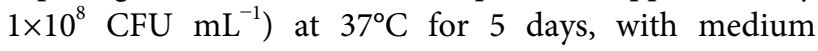
changed every two days. The scaffold-bacterium constructs were rinsed thrice and fixed in 95\% ethyl alcohol and the biomass of adhered biofilms was quantified by the crystal violet staining, as described previously [5]. In short, each scaffold was added with $1 \%(w / v)$ crystal violet at room temperature and kept for $15 \mathrm{~min}$. Excess dyes were removed and the scaffolds were flushed under tap water until no color appearing in washing stream. Optical photographs of the stained specimens were acquired. The biofilm-associated dyes were dissolved in $1 \mathrm{~mL}$ of $95 \%(v /$ $v$ ) ethanol under shaking at $37^{\circ} \mathrm{C}$ for $30 \mathrm{~min}$, followed by absorbance measurement for the eluates at $570 \mathrm{~nm}$.

\section{APTT, PT and TT tests}

APTT, PT and TT were tested to evaluate the influence of scaffolds on the local coagulation function. In detail, citrate anticoagulated human whole blood from a healthy volunteer supplied by Peking University Third Hospital was centrifuged at $3,000 \mathrm{rpm}$ for $15 \mathrm{~min}$ to separate the blood corpuscles, thus the platelet poor plasma (PPP) was prepared. And then the scaffold sample was immersed into $2 \mathrm{~mL}$ of PPP and incubated at $37^{\circ} \mathrm{C}$ for $30 \mathrm{~min}$, and subsequently the APTT, PT and TT were obtained using an Automatic coagulation analyzer (ACL TOP 700). Meanwhile, the blank PPP was tested as a control group.

\section{Cell proliferation on scaffold}

After cobalt-60 sterilization, the TS, TS-M, TS-M/P/V samples $(n=4)$ were seeded with hMSCs (Lonza, Walkersville) as described previously [8]. Generally, the samples were firstly immersed in culture medium for an hour, and then placed in 24-well plates (Ultra Low
Cluster Plate, Costar, Corning) before $50 \mu \mathrm{L}$ culture medium containing $1 \times 10^{5}$ cells was added. After that, Dulbecco's Modified Eagle's Medium (DMEM) containing $10 \%$ FBS and $1 \%$ penicillin/streptomycin were supplemented to the samples. The cells were cultured in an incubator at $37^{\circ} \mathrm{C}$ with $5 \% \mathrm{CO}_{2}$, with cultured medium refreshed twice per week. After incubating for 3, 7, and 14 days, the viability of cells cultured with the samples were assessed by the Cell Counting Kit (CCK-8; Dojindo, Japan). At each time point, the disks were rinsed thrice using PBS and incubated with $10 \%$ CCK- 8 solution at $37^{\circ} \mathrm{C}$ for $3 \mathrm{~h}$. The absorbance was measured at $450 \mathrm{~nm}$. Further, the amount and morphology of cells on the scaffolds were identified using SEM. For further investigating the cell viability on the scaffolds, we stained the live cells with Calcein AM and the dead cells with propidium iodide (LIVE/DEAD cell viability kit, Dojindo) and visualized the stained samples employing confocal laser scanning microscopy (CLSM).

\section{Alkaline phosphatase (ALP) activity}

Osteoblast differentiation ability was assayed in vitro by testing the ALP activity of hMSCs on the samples. Cell culture method and seeding protocol were the same as the one described above, except that the culture medium was replaced by the osteogenic one (OM, Lonza, Walkersville) containing $0.5 \%$ ascorbate, $0.5 \%$ dexamethasone, and $1 \%$ $\beta$-glycerophosphate for inducing osteogenic differentiation. After culturing the cells for 7 days, we detected the ALP activity employing the Alkaline Phosphatase Assay Kit (Beyotime, China). The cellular ALP activity was then normalized to the intracellular total protein level determined by a MicroBCA protein assay kit (Pierce).

\section{Statistical analysis}

All experiments were conducted in triplicate and expressed as mean values \pm standard deviations (SD). Statistical significance was analyzed by one-way variance analysis (ANOVA) or Student's t-test, and the level of significance was set at $p$ values less than 0.01 or 0.05 .

\section{RESULTS}

\section{Sample preparation and characterization}

In this study, custom designed 3D hierarchical porous Ti6Al4V biomaterials functionalized with vancomycin were realized by taking advantage of a facile MAO reaction and $\mathrm{PD} / \mathrm{Hep}$ assisted chemistry. The microstructure and surface morphology of scaffolds at different functionalization stages were studied by SEM. Firstly, mac- 

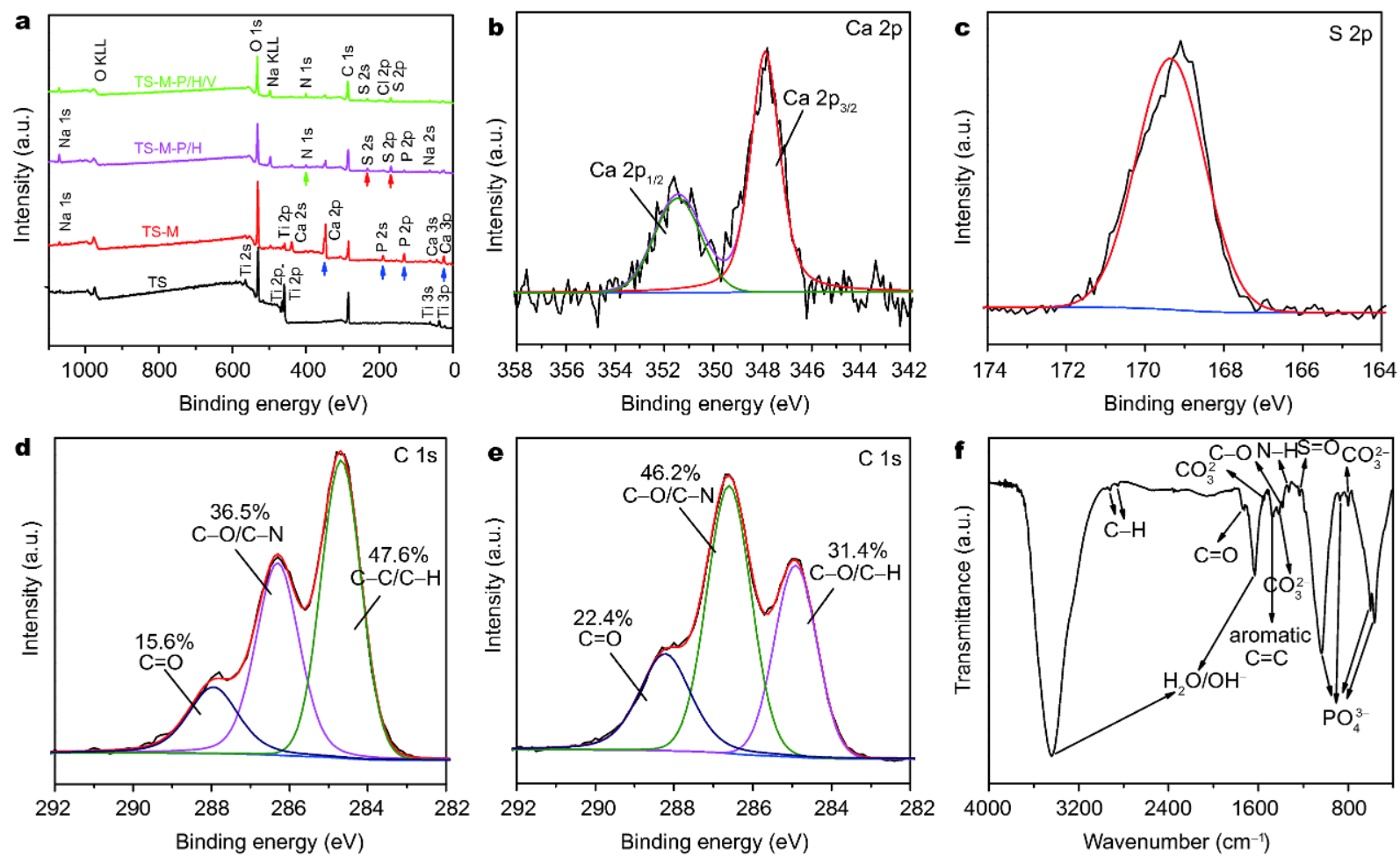

Figure 2 Chemical composition of the samples: (a) XPS survey spectra of TS (1), TS-M (2), TS-M/P/H (3), and TS-M/P/V (4); (b, c) the highresolution spectra of Ca $2 \mathrm{p}$ (b) and S $2 \mathrm{p}$ (c) for TS-M/P/V; (d, e) the high-resolution spectra of C 1s for TS-M/P/H (d) and TS-M/P/V (e); (f) representative FTIR spectrum of the TS-M/P/V scaffolds.

roporous TS constructs with hexagonal unit cells of pores (pore size $682 \mu \mathrm{m}$ ) were manufactured layer by layer via EBM (Fig. 1a). Secondly, $\sim 2-\mu$ m-thick substrate-adherent micro/nanoporous CaP coatings (pore sizes 0.1 to $2 \mu \mathrm{m}$ ) were in situ grown from titanium substrate via a rapid MAO process (Fig. 1b), as previously described [10]. The self-coating of a PD layer onto MAO, as well as the subsequent conjunction of heparin and vancomycin molecules relatively cover the primary porous surface landscapes (Fig. 1c).

Fig. 2a presents the XPS survey spectra of surface elements for scaffold samples at respective modification stages. On the TS, the major surface composition was identified as $\mathrm{Ti}, \mathrm{O}$ and $\mathrm{C}$, wherein the latter two were likely a result of oxidization and environmental contamination [5]. For TS-M, weakened substrate signals were witnessed while new peaks of $\mathrm{Ca}, \mathrm{P}$, and $\mathrm{Na}$ emerged, as thick $\mathrm{TiO}_{2}$ /apatite layers $(4-5 \mu \mathrm{m})$ were readily produced via MAO treatment on scaffold surface [10]. For TS-M/P/H and TS-M/P/V, the intensities for peaks of $\mathrm{Ca}$ and $\mathrm{P}$ decreased remarkably whilst peaks of substrate Ti became almost invisible. However, intensive new peaks of $\mathrm{N} 1 \mathrm{~s}, \mathrm{~S} 2 \mathrm{~s}$ and $\mathrm{S} 2 \mathrm{p}$ were observed in the spectrum, which, together with the strengthened $\mathrm{Na} 1 \mathrm{~s}$ signals (by heparin sodium), implicated that PD and heparin had been successfully introduced upon the MAO layers. Particularly, characteristic high resolution spectra of $\mathrm{Ca} 2 \mathrm{p}$ and $\mathrm{S} 2 \mathrm{p}$ were given in Fig. $2 \mathrm{~b}, \mathrm{c}$ as unique indicators of apatite and heparin, respectively. Compared with TS-M/P/H, a new peak at around $200 \mathrm{eV}$ attributable to $\mathrm{Cl} 2 \mathrm{p}$ was specifically detected for the $\mathrm{TS}-\mathrm{M} / \mathrm{P} / \mathrm{V}$, suggesting the possible existence of vancomycin. In addition, since the peak shape of $\mathrm{C} 1 \mathrm{~s}$ was obviously different for two sample types, their core-level spectra were acquired and fitted (Fig. 2d, e). Generally, C 1s core-level spectra for TS-M/P/H can be de-convoluted into three satellite peaks corresponding to various carbon bonding environments, namely $\mathrm{C}=\mathrm{O}$ at $288.0 \mathrm{eV}(15.6 \%), \mathrm{C}-\mathrm{O} / \mathrm{C}-$ $\mathrm{N}$ at $286.3 \mathrm{eV}(36.5 \%)$, and $\mathrm{C}-\mathrm{C} / \mathrm{C}-\mathrm{H}$ at $284.7 \mathrm{eV}(47.9 \%)$ $[38,39]$. The same spectral components were identified for TS-M/P/V. Nevertheless, the portions of $\mathrm{C}=\mathrm{O}$ and $\mathrm{C}-$ $\mathrm{O} / \mathrm{C}-\mathrm{N}$ bonds were increased significantly by $6.8 \%$ and 9.7\% respectively, while that of $\mathrm{C}-\mathrm{C} / \mathrm{C}-\mathrm{H}$ was decreased by $16.5 \%$. Notably, these data were in concert with the structural characteristics of heparin and vancomycin.

Furthermore, FTIR investigation was conducted for the 
TS-M-P/HV sample, as shown in Fig. 2f. The broad intense peaks observed at 3,441 and $1,631 \mathrm{~cm}^{-1}$ were associated with hydroxyl groups and adsorbed $\mathrm{H}_{2} \mathrm{O}$. The peaks at 2,924 and $2,853 \mathrm{~cm}^{-1}$ were attributed to $\mathrm{C}-\mathrm{H}$ vibration. The MAO induced growth of apatite coatings, as we previously reveled [10], was further corroborated by the typical peak of $\mathrm{PO}_{4}{ }^{3-}$ (triply degenerate $v 4$ vibration of $\mathrm{O}-\mathrm{P}-\mathrm{O}$ bonds) vibrations emerged at 562 and $604 \mathrm{~cm}^{-1}$, alongside with another two phosphate peaks at $874(\mathrm{vl}$ non-degenerate symmetric $\mathrm{P}-\mathrm{O}$ stretching band) and $1,041 \mathrm{~cm}^{-1}$ (triply degenerate $v 3$ antisymmetric $\mathrm{P}-\mathrm{O}$ stretching modes). Notably, the existence of $\mathrm{CO}_{3}^{2-}$ at around 1,551 (v1), 1,420 (v3) and $802 \mathrm{~cm}^{-1}$ (v2) implicates that $\mathrm{PO}_{4}{ }^{3-}$ lattice site is partially substituted by $\mathrm{CO}_{3}{ }^{2-}$ (carbonate-substituted apatites). Additionally, the peaks at 1,329 and $1,234 \mathrm{~cm}^{-1}$ correspond to the $\mathrm{N}-\mathrm{H}$ and $\mathrm{S}=\mathrm{O}$ antisymmetrical stretch vibrations, suggesting the successful immobilization of heparin mediated by polydopamine. Further, two characteristic peaks of vancomycin, i.e., aromatic $\mathrm{C}=\mathrm{C}\left(1,481 \mathrm{~cm}^{-1}\right)$ and $\mathrm{C}=\mathrm{O}$ $\left(1,726 \mathrm{~cm}^{-1}\right)$ stretching vibration bands were also identified [40], verifying the feasibility of this drug functionalization strategy. Combined with the results of XPS, it can be confirmed that vancomycin-loading composite coatings had already been constructed on porous titanium.

\section{Vancomycin release}

Release of vancomycin from TS-M/P/V and TS-P/V is investigated and shown in Fig. 3. The release process is composed of two time phases: burst release and slow stable release. In the first hour, $47.47 \%$ of vancomycin is released and then the release gradually decreases and shows a relatively stable release process over about 19 days. As shown in Fig. 3, release kinetics of vancomycin from TS-M/P/V went through a burst release (47.47\%) in the first hour. The left $43 \%$ is eluated in the rest $450 \mathrm{~h}$. In contrast, vancomycin release from TS-P/V has always remained low level, about $0.2 \mathrm{mg}$ throughout.

\section{In vitro antibacterial functions}

The initial antibacterial activity of the scaffolds was tested in vitro by incubating them with $S$. aureus $\left(1 \times 10^{8} \mathrm{CFU}\right.$ $\mathrm{mL}^{-1}$ ) for 4 and $24 \mathrm{~h}$. The antibacterial rates against the adhered (Raa) and planktonic (Rap) were determined and displayed in Fig. 4 . At $4 \mathrm{~h}$, the antibacterial rates of TS against the adhered (Raa) and planktonic (Rap) were negative values $(R \mathrm{aa}=-2.77 \%$, $R \mathrm{ap}=-3.68 \%)$, the $R \mathrm{aa}$ and Rap values for TS-M/P/V were as high as $95.66 \%$ and $94.41 \%$ respectively. The antibacterial rates at $24 \mathrm{~h}$ show similar results, implying excellent bactericidal ability

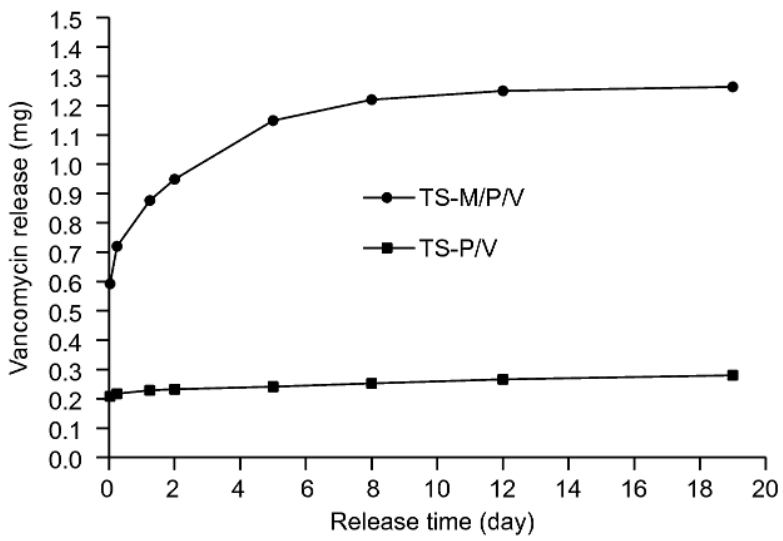

Figure 3 Release kinetics of vancomycin from TS-M/P/V and TS-P/V.

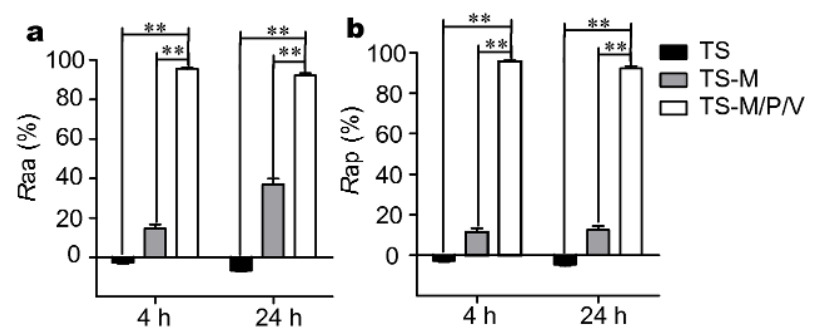

Figure 4 In vitro antibacterial activity against $S$. aureus on the surface (a) and in the surroundings (b) for 4 and $24 \mathrm{~h}(* * p<0.01)$.

against $S$. aureus both on surfaces and in surroundings. Additionally, the amount and morphology of adherent bacteria on scaffolds were investigated by SEM, as given in Fig. 5. Typical spherical S. aureus with intact membrane adhered preferentially onto TS and they had formed bacterium aggregates (red arrows, Fig. 5a, b) at $4 \mathrm{~h}$. For TS-M, it seemed bacterial adherence was not altered too much, but interestingly, adhesion mainly took place at the porous sunk sites (red arrows, Fig. $5 \mathrm{a}-\mathrm{d}$ ). By sharp contrast, no visible cells could be observed in TS$\mathrm{M} / \mathrm{P} / \mathrm{V}$, except for debris of disrupted bacterial membrane (red circles, Fig. 5e, f).

As cultivation went on to $24 \mathrm{~h}$, concentration of planktonic bacteria and adhered bacteria sonicated to solutions from samples were also detected by Microbial Viability Assay Kit-WST methods. The antibacterial rates of TS-M/P/V against the adhered (Raa; Fig. 4a) and planktonic bacteria (Rap; Fig. 4b), are $92.26 \%$ and $95.93 \%$, respectively, significantly higher than that of TS and TS-M, demonstrating the significant antibacterial and anti-adhesive efficiency of TS-M/P/V towards $S$. aureus for $24 \mathrm{~h}$ challenge.

In order to verify that the adherent bacteria were kil- 

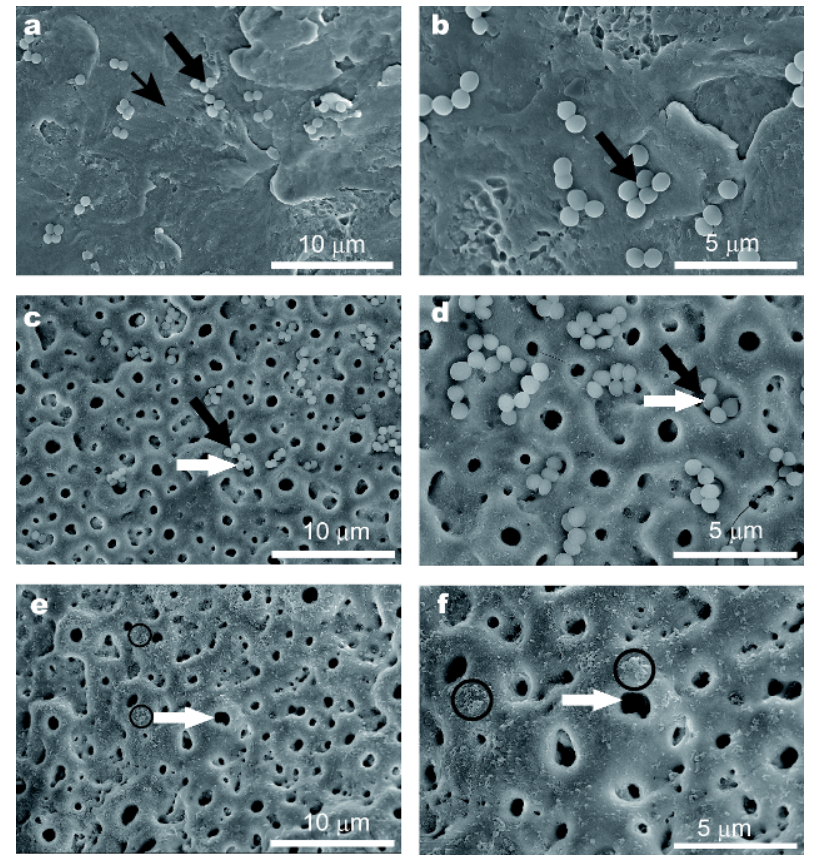

Figure 5 SEM images $(\times 4000$ and $\times 8000$ magnification) of samples with adhered S. aureus: TS (a, b); TS-M (c, d) and TS-M/P/V (e, f). In (c-f), white arrows indicate the micro/nanoporous produced by MAO; black arrows indicate the $S$. aureus colonizing within the pores. In (e, f), red circles indicate the debris of disrupted $S$. aureus membrane.

led, a CLSM-based Live/Dead fluorescent assay was performed after samples were co-cultured with $S$. aureus $\left(1 \times 10^{8} \mathrm{CFU} \mathrm{mL} \mathrm{m}^{-1}\right)$ for $24 \mathrm{~h}$, as demonstrated in Fig. 6 . Bacteria with intact and distored membranes were visualized green and red, respectively. Apparently, a wealth of living, green stained S. aureus existed on the surfaces and within scaffolds of TS and TS-M, ready to evolve into biofilms. By comparison, bacterial invasion was inhibited significantly on TS-M/P/V scaffolds, with most adherent bacteria dead and stained red.

Further, the cultivation was prolonged to 5 days for estimating the anti-biofilm activities of different scaffolds, and Gram's crystalviolet staining assay was carried out to determine the total amount of biomass (including the bacteria and the components of biofilms). Fig. $7 a-c$ depicted optical pictures of the tested scaffolds. Obviously, the TS and TS-M were featured by large areas of deep blue stains (mature biofilms), which coincided with previous results. Nevertheless, after vancomycin was introduced, the scaffolds became highly resistant to biofilm development, as little blue spots could be identified for the TS-M/P/V. Quantitatively, the biofilm-associated crystal violet stains were released and spectrochemically measured (Fig. 7d). Higher absorbance corresponded to stronger staining and thereby more accumulated biomass. The biomass for TS-M/P/V scaffolds was reduced remarkably by $52 \%$ and $63 \%$ compared to that of TS and TS-M respectively, confirming the beneficial effects of vancomycin release on eliminating bacterial growth and hence biofilm development.

\section{Anticoagulation potency}

The in vitro anticoagulation potency of the scaffolds was assessed using APTT, PT and TT tests, which have been extensively employed for evaluating the hemo-compatibility of various heparin treated biomaterials $[29,41]$. APTT and PT indicate the efficacy of intrinsic and extrinsic coagulation pathways, respectively, and TT is generally employed to test the activity of fibrinolytic systems. As shown in Fig. 8, the APTT, PT and TT values of TS-M/P/V were consistently prolonged compared to TS and TS-M $(p<0.05)$. The results implied that heparin maintained its anticoagulant properties during the whole process.

\section{Osteocompatibility and osteogenic activities}

For a bone substitute for mechanical bearing purpose, it is expected to facilitate favorable osteointegration at the implant/bone interface. In other words, the implant coatings should not only deter the adhesion and colonization of bacteria, but also encourage osteoblasts to adhere, proliferate and mature, so as to accomplish longterm stability of the implanted scaffolds. Fortunately, our in vitro cell research indicated that the TS-M/P/V had
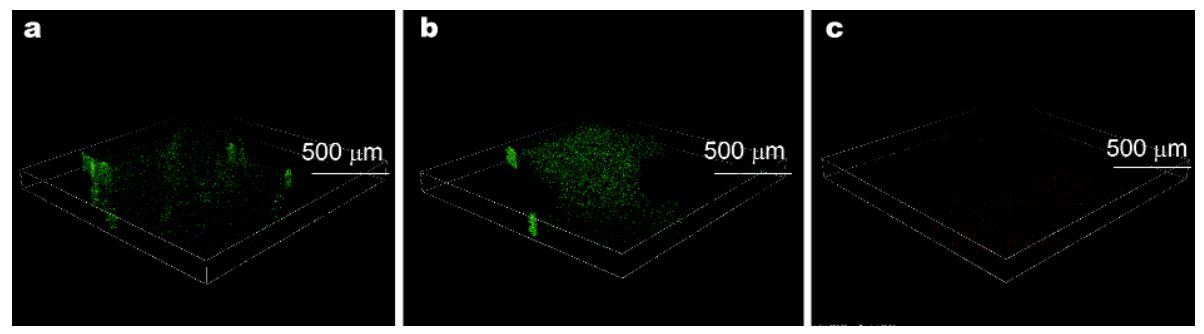

Figure 6 3D CLSM images of Live/Dead stained S. aureus on TS, TS-M and TS-M/P/V. Magnification is $\times 200$ and the scale bar is $500 \mu \mathrm{m}$. 

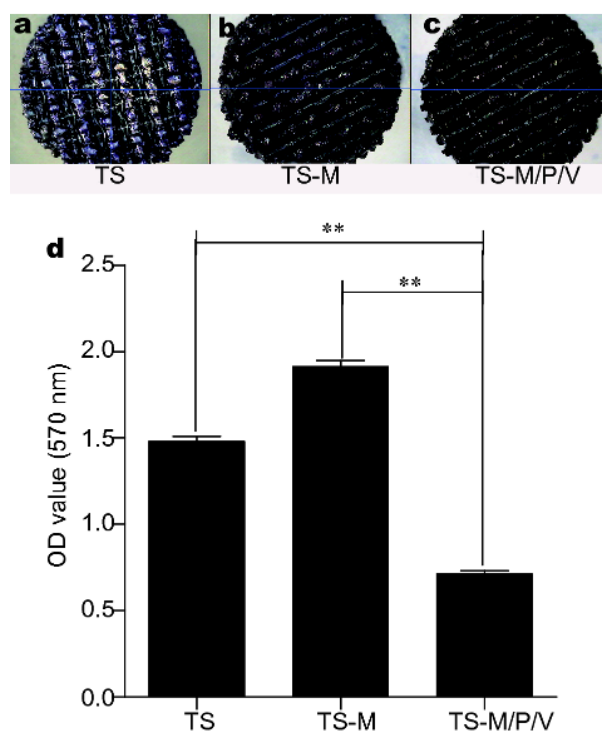

Figure 7 The biomass including the bacteria and the components of biofilms for different groups $(* * p<0.01)$ as visualized by crystal violet.

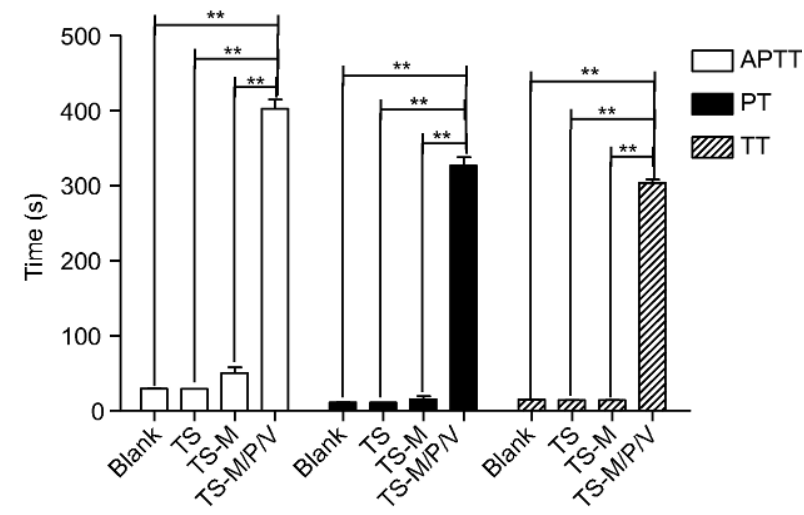

Figure 8 Clotting time test of different samples by APTT, PT and TT (mean $\pm \mathrm{SD}, n=6, * * p<0.01$ ).

good cytocompatibility with hMSCs.

Initially, we measured the proliferation of hMSCs on TS, TS-M and TS-M/P/V by testing the cellular metabolic activity at 1,7 , and 14 days (Fig. 9a). During the first week, there was no significant difference among the three groups $(p<0.05)$. On day 14 , the TS-M group showed significantly better proliferative activity, especially compared to the TS group $(p<0.05)$. The TS-M/P/V group also showed relatively better proliferative activity than the TS group. Moreover, the cell morphology and viability on day 14 were measured employing SEM and Live/Dead staining analysis (Figs 10 and 11). Generally, the hMSCs could survive on all of these scaffolds, as no dead cells

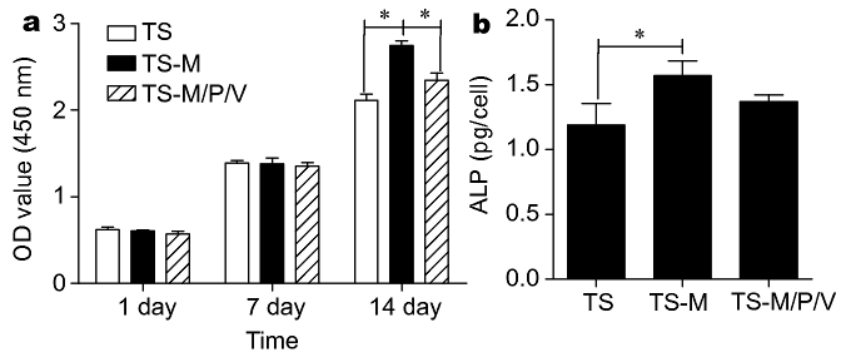

Figure 9 (a) Cell proliferation on the TS, TS-M and TS-M/P/V scaffolds $\left({ }^{*} p<0.05\right)$. (b) ALP activity of cells on the TS, TS-M and TS-M/P/V scaffolds after 7 days of culture $(* p<0.05)$.
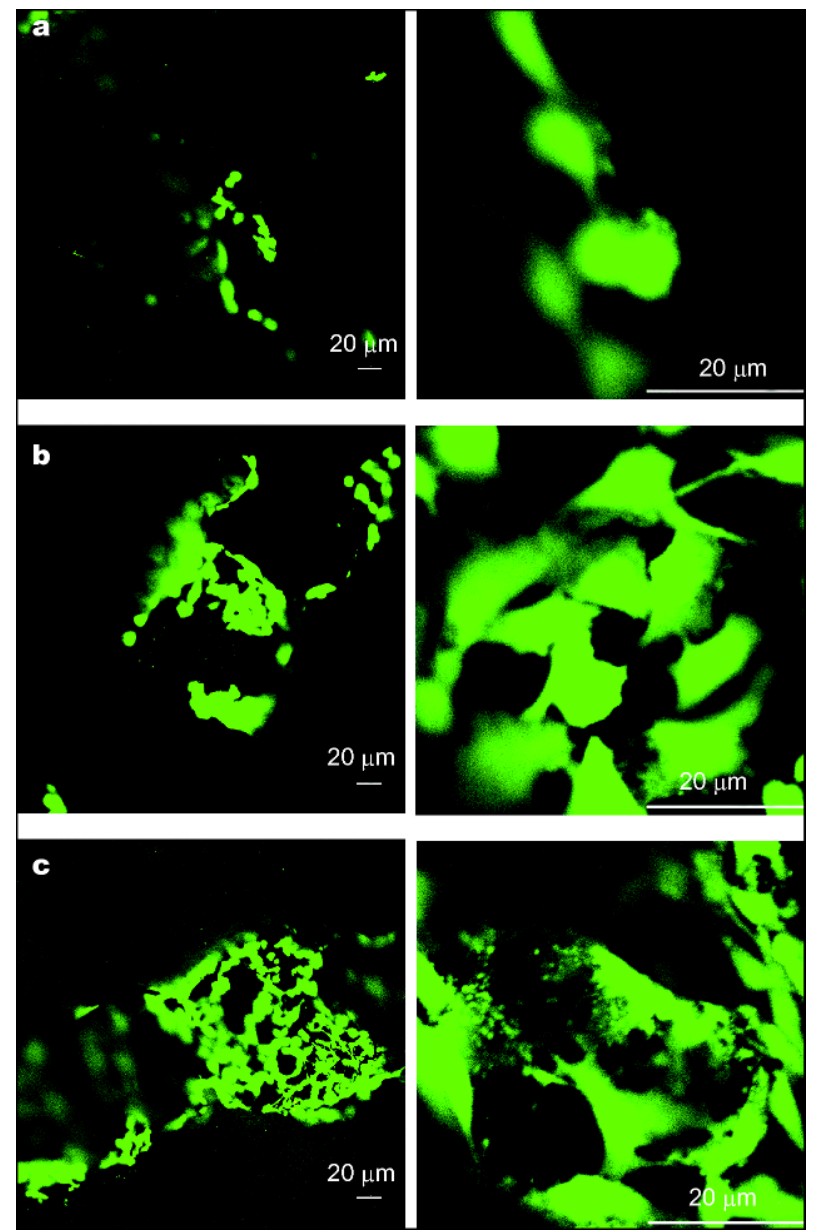

Figure 10 Live/Dead staining results of the TS (a), TS-M (b) and TSM/P/V (c) scaffolds after 14 days of cell culture. The right panels are magnified sections of the left. Live and dead cells appear green and red, respectively.

(red staining) could be observed. Clearly, the cell density on the TS-M and TS-M/P/V scaffolds was higher than that on the TS scaffold. Under SEM, the well-stretched hMSCs on TS-M and TS-M/P/V scaffolds presented fili- 

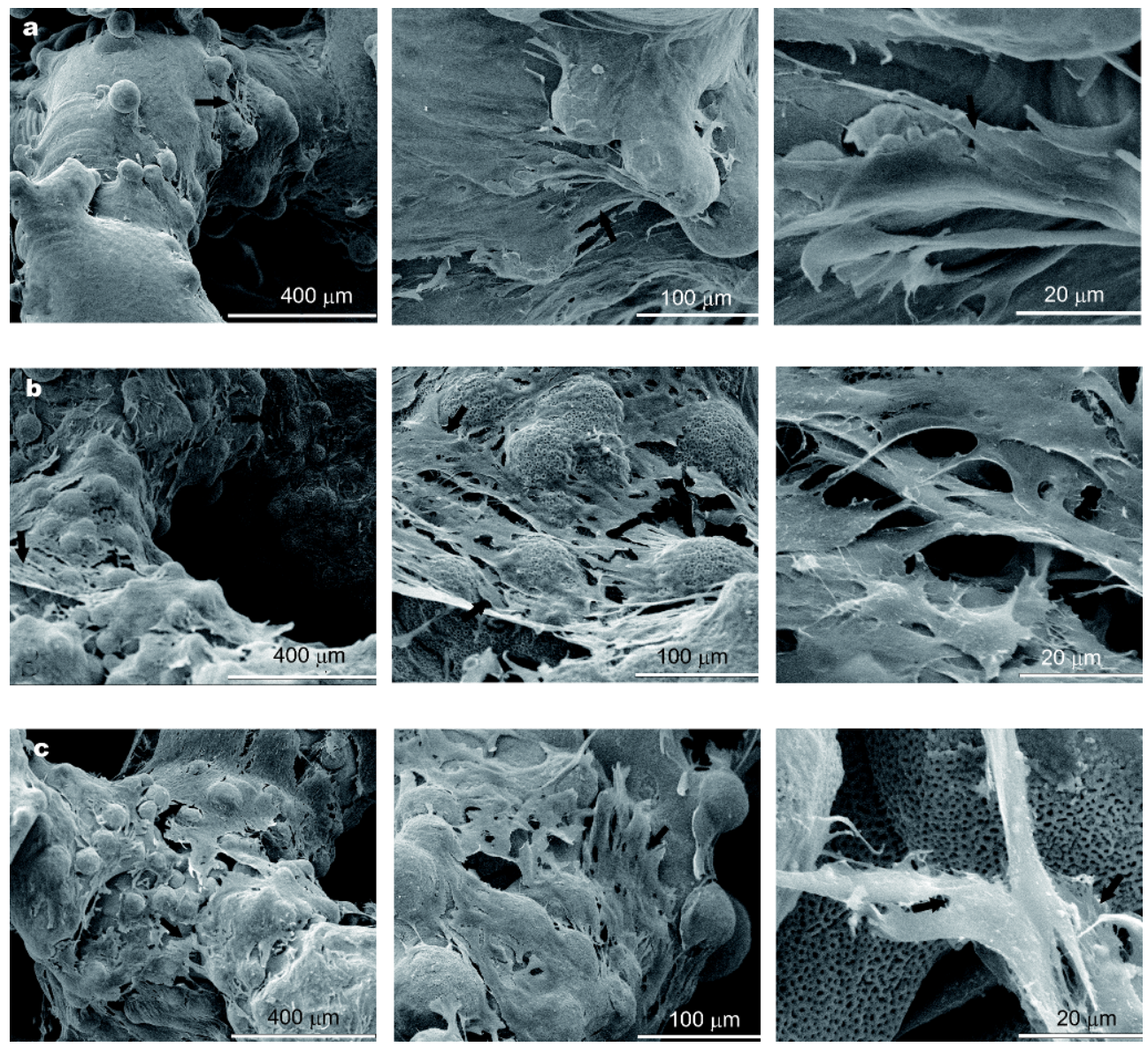

Figure 11 SEM observations of hMSCs on the TS (a), TS-M (b) and TS-M/P/V (c) scaffolds after 14 days of culture. Arrows point to cells.

form shapes and spread out more evenly on the substrate. More importantly, the hMSCs not only spread well on the surface but also extended to the metallic struts inside. Obviously, compared with the TS scaffolds, the amount and morphology of hMSCs on TS-M and TS-M/P/V scaffolds were both improved, which was consistent with the proliferation and staining analyses.

Furthermore, using ALP as an early phase osteogenetic marker, we measured the efficiency of our materials in promoting osteogenic differentiation of hMSCs. It was found that the TS-M and TS-M/P/V group presentedhigher ALP activity than the TS group on day 7 (Fig. 9b), indicating that the former two were more favorable to induce hMSC osteo-differentiation.

\section{DISCUSSION}

From perspectives of the orthopedists, a good orthopedic implant should possess the following merits: (1) Osteoconduction. It must be an ideal scaffold allowing for blood vessel ingrowth and new bone formation. (2) Stability. It should have sufficient mechanical strength for load-bearing and be anatomically matched to minimize the interfacial micromotions attributable to implant loosening. (3) Osteoinduction. It can enhance the proliferation and differentiation of hMSCs to promote osseointegration by means of providing biological active factors. (4) Antibacterial functions. While improving host cell adhesion, it can inhibit or kill bacteria that have the same adhesive mechanisms as host cells. Besides, favorable hemocompatibility and absorbability are also principal demands when the materials contact with blood [42]. 3D printed customized porous Ti6Al4V implants with interconnected macropores and high porosity can easily meet the requirements of osteoconduction and stability for its patient-specific design and bone in-growth properties $[43,44]$. In addition, the additive manufacturing technology has good flexibility, reproducibility and high cost-effectiveness. However, compared to conven- 
tional solid implants, porous implants with large specific surface area are more susceptible to bacterial colonization. But on the flip side of the coin, the large superficial area can be used as satisfactory substrate for endowing the scaffolds with multifunctionalities. In our previous study, micro/nanoporous apatite coatings were fabricated in situ on the alloy struts by MAO to improve the osseointegration of 3D printed porous Ti6Al4V implant [10]. Similarly, it is feasible to further decorate it with anti-bacterial and antiagglutinating functions through loading antimicrobials (antibiotics, silver, organic agents, bioactive molecules, etc.) and anticoagulants into these scaffolds simply by surfaces coatings.

Here, an original, feasible method for multiple functionalization of 3D printed Ti6Al4V scaffolds was proposed. First, TS was treated by MAO in the "electrolyte for osteointegrative coatings, ESOC", which creates micro-/nanopores $\mathrm{TiO}_{2} / \mathrm{CaP}$ coatings in situ on the TiS macroporous strut walls. In addition, electrolyte containing organic phosphate is proven to develop the biocompatibility of the MAO coating [45]. MAO has already been proven an efficient, convenient method suitable for the nanostructuring of macroporous surfaces [10]. Next, vancomycin and heparin were immobilized on the macro/nanoporous surfaces pre-modified assisted by a PDA layer, as originated from the self-polymerization of dopamine and bound tightly to $\mathrm{TiO}_{2}$ [22].The self-polymerized PDA layer is stable and biocompatible, but has poor blood compatibility, which restricts the clinically application to some extent. Heparin has been widely used for improving the blood compatibility of implant coating in clinic and possesses tremendous advantages, such as anti-inflammatory properties, significant effects on cell proliferation, and promotion of angiogenesis. The $-\mathrm{NH}-$ groups in heparin molecular chains could form covalent bonds with the quinone groups in PDA, thus heparin tightly anchored onto Ti surfaces [46]. Vancomycin, one of the most widely used antibiotics for prevention and treatment of postoperative orthopaedic infections, was charged and delivered through multiple interactions with PDA/heparin for sustained controlled release, which endowed our implants with excellent antibacterial and antibiofilm functions.

In our study, the improvement in cell attachment, proliferation and differentiation might originate from synergistic effects of the surface topography and chemical composition. Both micro-/nanoporous topography of $\mathrm{MAO}$ and the functional groups of PDA and heparin are previously shown to direct protein adsorption and cell adhesion and further promote osteoblast spreading, mi- gration and proliferation [5]. Further, the release of $\mathrm{Ca}^{2+}$ from the scaffold into the extracellular environment is beneficial to promoting the osteogenic differentiation of hMSCs on the scaffold [47]. And the higher ALP activity, an early phase indicator of the osteoblastic differentiation of hMSCs, exhibited by the treated group, demonstrates this point.

Recently, advanced orthopedic porous implants by 3D printing targeted at functional bone defect restoration have become a hot research topic; however, the focus is mainly on endowing them with osteointegration functions rather than antimicrobial activities $[13,48,49]$. To impart antimicrobial coatings onto orthopedic implants is an efficient and versatile approach. Generally, these coatings can be sorted into two types: inorganic and organic antimicrobial coatings. As a most widely used inorganic antimicrobial agent, nanosilver has been loaded into $\mathrm{TiO}_{2}$ micronanopores [5] and nanotubes [4] and dispersed into graphene oxide coatings [50], which endows bioceramic or biometallic scaffolds with strong bacteria elimination potencies. Nanosilver-based implant coatings possess advantages such as excellent antibacterial efficiency, good stability, and low occurrence of drug resistance. Nonetheless, the release profiles of silver should be strictly controlled, otherwise it will have adverse effects on the proliferation of human cells. On the other hand, organic antimicrobial coatings are alternatively applied to 3D printing orthopedic implants, such as quaternized chitosan-PLGA/HA scaffolds [51]. Of particular research interest are antibiotic eluting polymer coatings which hold great clinical translational potential. However, the drug loading efficiency is limited and duration of antibiotic release from commonly available polymer coatings is several hours to a few days [52,53]. In fact, constant aseptic condition is critical for normal osseous functions in the early healing stage (3 months). Hence, high drug-loading amount and sustained drug release are urgent unmet need for 3D-printed drug eluting implants. In the present study, vancomycin was loaded into the external and internal surfaces of porous titanium as treated by MAO and functionalized by PDA/ heparin, both of which were simple and facile processes. The high loading property and sustained delivery characteristics were confirmed in Fig. 3. This was likely enabled by the mutual contribution of largely enhanced specific surface areas (due to scaffold's macropores and coating's micro/nanopores) and versatile surface chemistry [5]. Consequently, appreciable antibacterial and antibiofilm performances were achieved against $S$. aureus (Figs 4-8), implicating good potential for the scaffolds' 
long term infection prophylaxis in real application. Notably, clinically relevant antibiotic vancomycin was selected and used, which was proved highly effective in impeding bacterial wall synthesis and killing most pathogenic bacteria. Up to date, it has shown no crossresistance to any other antibiotics and bacteria are hard to develop resistance for it. It should also be mentioned that the as-prepared scaffolds were stable for months at room temperature and did not lose their antibacterial potency (data not shown), indicating their sound shelf-life important in clinical practice.

Therefore, the multifunctionality of $\mathrm{TS}-\mathrm{M} / \mathrm{P} / \mathrm{V}$ was easily realized, especially the anti-infection and osteointegration, by utilizing the merits of the micro/nanoporous topology of MAO, the bioadhesion and biofunctionality of PDA, the hemo-compatibility of heparin, and further the antibacterial activity of vancomycin. In addition, thanks to the excellent 3D surface adaptability of MAO and PDA, both the external and interpores surfaces can be uniformly functionalized, boding well for future applications for treating complexed medical devices.

\section{CONCLUSIONS}

In this work, we have successfully prepared anti-infection and osteogenic multi-functional 3D printed porous Ti6Al4V implants with excellent hemo-compatibility by MAO together with loading vancomycin and heparin and further investigated the vancomycin release kinetic, antiinfection and osteogenic capacity, cytocompatibility and hemocompatibility. The MAO treatment greatly increased the vancomycin releasing amount, which can reach $1.26 \mathrm{mg}$ within 19 days. In comparison with blank control group, the treatment group displayed a remarkable enhancement in anti-infection and osteogenic capacity. Furthermore, the treatment group exhibited good cytocompatibility and hemocompatibility. In total, this multi-functional 3D printed porous Ti6Al4V implant can play a role in customized functional reconstruction of intricate bone defects.

Received 27 October 2017; accepted 4 January 2018; published online 7 February 2018

1 Yang F, Chen C, Zhou QR, et al. Laser beam melting 3D printing of Ti6Al4V based porous structured dental implants: fabrication, biocompatibility analysis and photoelastic study. Sci Rep, 2017, 7: 45360

2 Shah FA, Snis A, Matic A, et al. 3D printed Ti6Al4V implant surface promotes bone maturation and retains a higher density of less aged osteocytes at the bone-implant interface. Acta Biomater,
2016, 30: 357-367

3 Medicrea International. French Surgeon Performs World's First Spinal Fusion Surgery Using Customized 3-D Printed Spine Cages. Business Wire (English), 2014

4 Amin Yavari S, Loozen L, Paganelli FL, et al. Antibacterial behavior of additively manufactured porous titanium with nanotubular surfaces releasing silver ions. ACS Appl Mater Interfaces, 2016, 8: 17080-17089

5 Jia Z, Xiu P, Xiong P, et al. Additively manufactured macroporous titanium with silver-releasing micro-/nanoporous surface for multipurpose infection control and bone repair-a proof of concept. ACS Appl Mater Interfaces, 2016, 8: 28495-28510

6 Busscher HJ, van der Mei HC, Subbiahdoss G, et al. Biomaterialassociated infection: locating the finish line in the race for the surface. Sci Transl Med, 2012, 4: 153rv10-153rv10

7 Saleh J, El-Othmani MM, Saleh KJ. Deep vein thrombosis and pulmonary embolism considerations in orthopedic surgery. Orthop Clin North Am, 2017, 48: 127-135

8 Lv J, Jia Z, Li J, et al. Electron beam melting fabrication of porous Ti6Al4V scaffolds: cytocompatibility and osteogenesis. Adv Eng Mater, 2015, 17: 1391-1398

9 Lopez-Heredia MA, Sohier J, Gaillard C, et al. Rapid prototyped porous titanium coated with calcium phosphate as a scaffold for bone tissue engineering. Biomaterials, 2008, 29: 2608-2615

10 Xiu P, Jia Z, Lv J, et al. Tailored surface treatment of 3D printed porous Ti6Al4V by microarc oxidation for enhanced osseointegration via optimized bone in-growth patterns and interlocked bone/implant interface. ACS Appl Mater Interfaces, 2016, 8: 17964-17975

11 Amin Yavari S, van der Stok J, Chai YC, et al. Bone regeneration performance of surface-treated porous titanium. Biomaterials, 2014, 35: 6172-6181

12 Lee H, Jang TS, Song J, et al. Multi-scale porous Ti6Al4V scaffolds with enhanced strength and biocompatibility formed via dynamic freeze-casting coupled with micro-arc oxidation. Mater Lett, 2016, 185: $21-24$

13 Radtke A, Topolski A, Jędrzejewski T, et al. The bioactivity and photocatalytic properties of titania nanotube coatings produced with the use of the low-potential anodization of Ti6Al4V alloy surface. Nanomaterials, 2017, 7: 197

14 Cao H, Liu X. Plasma-sprayed ceramic coatings for osseointegration. Int J Appl Ceram Technol, 2013, 10: 1-10

15 Govindharajulu JP, Chen X, Li Y, et al. Chitosan-recombinamer layer-by-layer coatings for multifunctional implants. Int J Mol Sci, 2017, 18: 369

16 Zhou L, LV GH, Mao FF, Yang SZ. Preparation of biomedical Ag incorporated hydroxyapatite/titania coatings on Ti6Al4V alloy by plasma electrolytic oxidation. Chin Phys, 2014, 3: 035205

17 Darouiche RO. Device-associated infections: a macroproblem that starts with microadherence. Clin Infect Dis, 2001, 33: 1567-1572

18 Zimmerli W, Waldvogel FA, Vaudaux P, et al. Pathogenesis of foreign body infection: description and characteristics of an animal model. J Infect Dis, 1982, 146: 487-497

19 Ceri H, Olson ME, Stremick C, et al. The Calgary Biofilm Device: New technology for rapid determination of antibiotic susceptibilities of bacterial biofilms. J Clin Microbiol, 1999, 6: 1771-1776

20 Ishibe $\mathrm{T}$, Goto $\mathrm{T}$, Kodama $\mathrm{T}$, et al. Bone formation on apatitecoated titanium with incorporated BMP-2/heparin in vivo. Oral Surgery Oral Med Oral Pathol Oral Rad Endodontol, 2009, 108: $867-875$ 
21 Lee DW, Yun YP, Park K, et al. Gentamicin and bone morphogenic protein-2 (BMP-2)-delivering heparinized-titanium implant with enhanced antibacterial activity and osteointegration. Bone, 2012, 50: 974-982

22 Kim SE, Song SH, Yun YP, et al. The effect of immobilization of heparin and bone morphogenic protein-2 (BMP-2) to titanium surfaces on inflammation and osteoblast function. Biomaterials, 2011, 32: 366-373

23 Teixeira S, Yang L, Dijkstra PJ, et al. Heparinized hydroxyapatite/ collagen three-dimensional scaffolds for tissue engineering. J Mater Sci-Mater Med, 2010, 21: 2385-2392

24 Schroeder-Tefft JA, Bentz H, Estridge TD. Collagen and heparin matrices for growth factor delivery. J Control Release, 1997, 48: 29-33

25 Meneghetti MCZ, Hughes AJ, Rudd TR, et al. Heparan sulfate and heparin interactions with proteins. J R Soc Interface, 2015, 12: 20150589

26 Sofroniadou S, Revela I, Smirloglou D, et al. Linezolid versus vancomycin antibiotic lock solution for the prevention of nontunneled catheter-related blood stream infections in hemodialysis patients: a prospective randomized study. Seminars Dialysis, 2012, 25: $344-350$

27 Cesaro S, Cavaliere M, Spiller M, et al. A simplified method of antibiotic lock therapy for Broviac-Hickman catheters using a CLC 2000 connector device. Support Care Cancer, 2007, 15: 95-99

28 Moore CL, Besarab A, Ajluni M, et al. Comparative effectiveness of two catheter locking solutions to reduce catheter-related bloodstream infection in hemodialysis patients. Clinical J Am Soc Nephrology, 2014, 9: 1232-1239

29 Wu C, Han P, Liu X, et al. Mussel-inspired bioceramics with selfassembled Ca-P/polydopamine composite nanolayer: Preparation, formation mechanism, improved cellular bioactivity and osteogenic differentiation of bone marrow stromal cells. Acta Biomater, 2014, 10: 428-438

30 Cheng C, Li S, Zhao W, et al. The hydrodynamic permeability and surface property of polyethersulfone ultrafiltration membranes with mussel-inspired polydopamine coatings. J Membrane Sci, 2012, 417-418: 228-236

31 Lee H, Dellatore SM, Miller WM, et al. Mussel-inspired surface chemistry for multifunctional coatings. Science, 2007, 318: 426430

32 Kang SM, Hwang NS, Yeom J, et al. One-step multipurpose surface functionalization by adhesive catecholamine. Adv Funct Mater, 2012, 22: 2949-2955

33 Lee H, Rho J, Messersmith PB. Facile conjugation of biomolecules onto surfaces via mussel adhesive protein inspired coatings. Adv Mater, 2009, 21: 431-434

34 Jia Z, Shi Y, Xiong P, et al. From solution to biointerface: graphene self-assemblies of varying lateral sizes and surface properties for biofilm control and osteodifferentiation. ACS Appl Mater Interfaces, 2016, 8: 17151-17165

35 Jiang JH, Zhu LP, Li XL, et al. Surface modification of PE porous membranes based on the strong adhesion of polydopamine and covalent immobilization of heparin. J Membrane Sci, 2010, 364: 194-202

36 Zhu LP, Yu JZ, Xu YY, et al. Surface modification of PVDF porous membranes via poly(DOPA) coating and heparin immobilization. Colloids Surfs B-Biointerfaces, 2009, 69: 152-155

37 Lv J, Xiu P, Tan J, et al. Enhanced angiogenesis and osteogenesis in critical bone defects by the controlled release of BMP-2 and VEGF: implantation of electron beam melting-fabricated porous Ti6Al4 scaffolds incorporating growth factor-doped fibrin glue. Biomed Mater, 2015, 10: 035013

38 Del Frari D, Bour J, Ball V, et al. Degradation of polydopamine coatings by sodium hypochlorite: A process depending on the substrate and the film synthesis method. Polymer Degradation Stability, 2012, 97: 1844-1849

39 Ma L, Cheng C, He C, et al. Substrate-independent robust and heparin-mimetic hydrogel thin film coating via combined LbL selfassembly and mussel-inspired post-cross-linking. ACS Appl Mater Interfaces, 2015, 7: 26050-26062

40 Ordikhani F, Tamjid E, Simchi A. Characterization and antibacterial performance of electrodeposited chitosan-vancomycin composite coatings for prevention of implant-associated infections. Mater Sci Eng-C, 2014, 41: 240-248

41 Liu T, Liu Y, Chen Y, et al. Immobilization of heparin/poly-l-lysine nanoparticles on dopamine-coated surface to create a heparin density gradient for selective direction of platelet and vascular cells behavior. Acta Biomater, 2014, 10: 1940-1954

42 Liu Y, Zheng Y, Hayes B. Degradable, absorbable or resorbablewhat is the best grammatical modifier for an implant that is eventually absorbed by the body? Sci China Mater, 2017, 60: 377391

43 Yang J, Cai H, Lv J, et al. In vivo study of a self-stabilizing artificial vertebral body fabricated by electron beam melting. Spine, 2014, 39: E486-E492

$44 \mathrm{Xu} \mathrm{N}$, Wei F, Liu X, et al. Reconstruction of the upper cervical spine using a personalized 3D-printed vertebral body in an adolescent with Ewing sarcoma. Spine, 2016, 41: E50-E54

45 Zhang RF, Qiao LP, Qu B, et al. Biocompatibility of micro-arc oxidation coatings developed on Ti6Al4V alloy in a solution containing organic phosphate. Mater Lett, 2015, 153: 77-80

46 Li G, Xie B, Pan C, et al. Facile conjugation of heparin onto titanium surfaces via dopamine inspired coatings for improving blood compatibility. J Wuhan Univ Technol-Mat Sci Edit, 2014, 29: 832840

47 Barradas AMC, Fernandes HAM, Groen N, et al. A calcium-induced signaling cascade leading to osteogenic differentiation of human bone marrow-derived mesenchymal stromal cells. Biomaterials, 2012, 33: 3205-3215

48 Bouyer M, Guillot R, Lavaud J, et al. Surface delivery of tunable doses of BMP-2 from an adaptable polymeric scaffold induces volumetric bone regeneration. Biomaterials, 2016, 104: 168-181

49 Inzana JA, Olvera D, Fuller SM, et al. 3D printing of composite calcium phosphate and collagen scaffolds for bone regeneration. Biomaterials, 2014, 35: 4026-4034

50 Xu S, Song J, Zhu C, et al. Graphene oxide-encapsulated Ag nanoparticle-coated silk fibers with hierarchical coaxial cable structure fabricated by the molecule-directed self-assembly. Mater Lett, 2017, 188: 215-219

51 Yang Y, Yang S, Wang Y, et al. Anti-infective efficacy, cytocompatibility and biocompatibility of a 3D-printed osteoconductive composite scaffold functionalized with quaternized chitosan. Acta Biomater, 2016, 46: 112-128

52 Pérez-Anes A, Gargouri M, Laure W, et al. Bioinspired titanium drug eluting platforms based on a poly- $\beta$-cyclodextrin-chitosan layer-by-layer self-assembly targeting infections. ACS Appl Mater Interfaces, 2015, 7: 12882-12893

53 Önder S. Surface modification of titanium using bsa-loaded chitosan and chitosan/gelatin polymers. J Med Biol Eng, 2016, 36: 661-667 
Acknowledgements The authors acknowledge the Grant from Ministry of Science and Technology of China (2016YFB1101501) and research and financial support from the Beijing AKEC Medical Co., Ltd. and Medical Research Center of Peking University Third Hospital.

Author contributions Zhang $\mathrm{T}$ and Zhou $\mathrm{W}$ designed the research; Liu Z, Zheng Y, and Cheng Y supervised the project; Zhou W, Yan J and Fan $\mathrm{D}$ performed the experiments, Zhang $\mathrm{T}$ and Jia $\mathrm{Z}$ prepared the manuscript; Jia Z, Yin C, Wei Q, Cai H, Liu X and Zhou H contributed in discussion, language improvements, and proof reading; Yang $\mathrm{X}$ designed and fabricated the scaffolds.

Conflict of interest The authors declare no conflict of interest.

Supplementary information Supporting information is available in the online version of the paper.

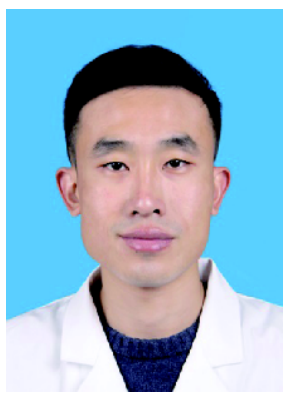

Teng Zhang is currently a PhD student at Peking University Health Sciencce Center, under the supervision of Prof. Zhongjun Liu. His research interests include the 3D printed orthopaedic implants and its surface modification.

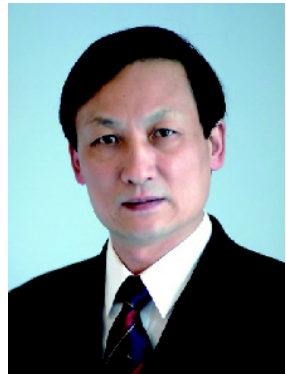

Zhongjun Liu is the Chair of the Department of Orthopaedic Surgery in Peking University Third Hospital, the Leader of Orthopaedic Innovating Team of Ministry of Education, China, the consultant of central health care, and the pioneer in clinical application of 3D printing spinal implants and related research.

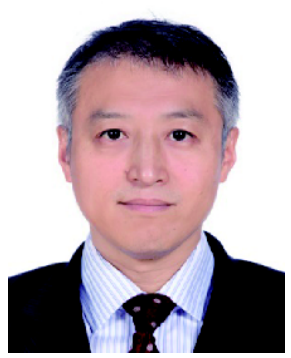

Yufeng Zheng received his PhD in materials science from Harbin Institute of Technology, China, in 1998. Since 2004, he has been a full professor at Peking University in Beijing, China. His research focuses on the development of various new biomedical metallic materials (biodegradable $\mathrm{Mg}, \mathrm{Fe}$ and $\mathrm{Zn}$ based alloys, $\beta$-Ti alloys with low elastic modulus, bulk metallic glass, ultra-fine grained metallic materials, etc.).

\section{聚多巴胺辅助微弧氧化后载肝素和万古霉素以提高3D打印多孔钛合金内植物的血液相容性和抗菌成 骨功能}

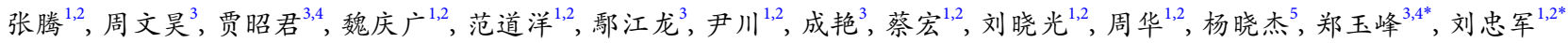

摘要 在研发多功能骨科生物材料时, 抗菌能力、血液相容性、骨细胞相容性和骨整合性能是最重要的考虑因素. 本文中, 我们成功研发 了具有良好血液相容性的抗菌、促成骨复合功能的3D打印多孔钛合金内植物. 简言之, 3D打印多孔钛合金支架经表面处理后具有多孔形 貌的微弧氧化促成骨涂层, 进而联结固定肝素钠和万古霉素. 本文采用扫描电镜、X射线光电子能谱分析和傅里叶变换红外光谱学对其表 面微观结构、形态和化学组成进行了表征, 并用高效液相色谱法检测了其载药能力和万古霉素释放曲线. 通过微生物检测、细菌死活染 色和结晶紫染色, 确定了其良好的抗金葡菌及菌膜性能. 将人骨髓间充质干细胞在支架上培养后, 用细胞计数试剂盒和细胞活性检测试剂 盒检测了其增殖活性. 此外, 用碱性磷酸酶成活性作为标记检测了成骨细胞分化能力, 通过检测活化部分凝血活酶时间、血浆凝血酶原时 间和凝血酶时间, 确定肝素化的支架血液相容性得到了提高. 该研究结果表明, 经过表面改性的3D打印多孔钛合金内植物拥有稳定的抗菌 和促成骨功能, 同时显现出抗凝特性. 预示着其在未来能用于复杂骨缺损的定制化功能重建. 